\title{
Endorsement of the HerniaSurge guidelines by the Asia Pacific Hernia Society
}

\author{
A. Sharma ${ }^{1}$
}

Received: 13 July 2017 / Accepted: 20 September 2017 / Published online: 12 January 2018

(c) Springer-Verlag France SAS 2017

The APHS wishes to congratulate all stakeholders involved in the creation and preparation of HerniaSurge guidelines, the International Guidelines for groin hernia management. I believe these guidelines are the most elaborate, broad-based and comprehensive guidelines on groin hernia management that have been created. The Asia Pacific Hernia Society is happy to have been associated and contributed.

HerniaSurge guidelines are comprehensive (open and laparoscopy) and address relevant issues such as indications, preparations, operating room layout, techniques of choice, special situations, complications and economics. Each topic is structured and presented in a manner that facilitates ease of reading and is comprehensive. All relevant statements are discussed in detail in the "Discussion" section incorporating currently available levels of evidence. The scientific process has been meticulous and rigorous, including the final ratification by external reviewers, according to the AGREE II guidelines.

We expect HerniaSurge guidelines to be consulted and referenced by both the "Maestro" and the "Tyro" involved in hernia care and management. The APHS currently offers a structured training program in hernia called "Hernia Essentials" based on current international guidelines on hernia management. Recommendation and statements from HerniaSurge guidelines are to be extensively quoted in this training program.

Perhaps, the most telling comment was from our Fellow in Minimal access surgery, who said "I found it most useful to refer to the current version of HerniaSurge guidelines for all my clinical, research and publication requirements for hernia".

A. Sharma, President APHS.

Compliance with ethical standards

Conflict of interest The author declares that there is no conflict of interest.

This comment refers to the article available at doi:10.1007/ s10029-017-1668-x.

A. Sharma

anil.sharma@maxhealthcare.com

1 Max Super Specialty Hospital, Saket, New Delhi, India 